\title{
Fibromyalgia, myofascial pain, tender points and trigger points: splitting or lumping?
}

\author{
Robert M Bennett*1 and Don L Goldenberg ${ }^{2}$ \\ See related research by Ge et al., http://arthritis-research.com/content/13/2/R48
}

\begin{abstract}
Myofascial trigger points (MTPs) have long been a contentious issue in relation to fibromyalgia, and poorly defined pain complaints in general. Can MTPs be reproducibly identified? Do MTPs have valid objective findings, such as spontaneous electromyographic activity, muscle microdialysis evidence for an inflammatory milieu or visualization with newer ultrasound techniques? Is fibromyalgia a syndrome of multiple MTPs, or is focal muscle tenderness a manifestation of central sensitization? These issues are discussed with relevance to a recent paper reporting that manual palpation of active MTPs elicits the spontaneous pain experienced by fibromyalgia patients.
\end{abstract}

\section{Point: Dr Bennett}

The paper by Ge and colleagues from the Center for Sensory-Motor Interaction at Aalborg University, Denmark provides evidence that peripheral nociceptive input from muscle may be relevant to the contemporary understanding of fibromyalgia (FM) [1].

This study involved asking each subject (both FM patients and controls) to draw all areas of current spontaneous pain on an anatomical map and rate the overall intensity of pain. The area of pain was quantified by digitization software. The location of all active myofascial trigger points (MTPs) was then determined in the FM subjects using clinical palpation [2]. Altogether 308 active MTPs were found in the 30 FM subjects, and 305 of these were confirmed by the demonstration of spontaneous electrical activity on needle electromyography (EMG). The locations of these 308 active MTPs were then

*Correspondence: bennetrob1@comcast.net

'Fibromyalgia Research Unit, Oregon Health \& Science University, 3455 SW

Veterans Road, Portland, OR 97239, USA

Full list of author information is available at the end of the article mirrored onto the 30 healthy controls as an aid to identifying latent MTPs; spontaneous electrical activity was found in 304 of these latent MTPs. The major MTP in each muscle was manually palpated at a pressure of about $4 \mathrm{~kg}$ for 10 seconds, and the location and area of referred pain was drawn by the subject and later digitized for subsequent analysis.

The major findings were as follows. The intensity of the spontaneous pain in FM was strongly correlated with the total area of pain referred by manual palpation of MTPs. Manual stimulation of active MTPs in FM produces a local and referred pain pattern that is similar to the subject's current spontaneous pain report. The locations of active MTPs in FM subjects were generally found to be the site of latent MTPs in the controls. The overall number of MTPs was similar in both the FM patients and control subjects, but FM subjects had active MTPs whereas the controls' MTPs were latent. Active MTPs in the FM subjects were most commonly found in the extensor digitorum, trapezius and infraspinatus in the upper body, and in the quadratus lumborum and gluteus medius in the lower body.

A critical issue in understanding Ge and colleagues' paper is the distinction between active and latent MTPs. Ge and colleagues used the Travell and Simons recommendations for finding a MTP [2]; these specify that gentle palpation should be performed across the direction of the muscle fibers in order to identify a region of tenderness and nodularity (that is, the taut band). Continued firm palpation of a MTP for at least 5 seconds is required to elicit the typical distribution of referred pain. An active MTP is deduced if firm pressure over the taut band reproduces the patient's spontaneous pain symptoms. If the pain symptoms are not reproduced, the tender area is designated a latent trigger point. Latent MTPs are a common finding in healthy individuals, as is evident to anyone who has ever had a therapeutic massage.

The Aalborg research group has a long record of productive research in the area of myofascial pain (MFP) and has recently presented evidence that most of the 18 tender points used in the 1990 classification criteria for FM have the characteristics of MTPs [3]. Over the past 
two decades, clinicians have often observed or hypothesized a role for MTPs in the pathogenesis of FM [4-6]. The lack of any generally acceptable criteria for reproducibly locating MTPs has dissuaded many researchers from pursuing this avenue of investigation [7]. In the past 5 years, however, there have been several studies that have provided a better scientific underpinning for understanding MTPs [8]: microdialysis has shown that MTPs have an acidic milieu containing pro-nociceptive molecules; MTPs can be visualized as a hypoechogenic area using specialized ultrasound techniques; MTPs have been visualized with magnetic resonance elastography; the stimulation of MTPs may lead to central sensitization; stimulation of MTPs evokes activation of brain locations that have been associated with pain and emotional processing; and insertion of a concentric electrode into a MTP results in spontaneous electrical activity that can be visualized on EMG.

Currently FM is envisaged to be a pain syndrome related to dysfunctional central pain processing; however, increasingly evident is that peripheral pain generators such as painful joints and MTPs now need to be incorporated into this model [9]. A more widespread acceptance of MTPs and other peripheral pain generators as potential initiators and perpetuators of central sensitization would be an important paradigm shift in our current understanding of FM. The relevance of MTPs is gaining increasing attention, and $\mathrm{Ge}$ and colleagues' results have now been replicated in a study from Spain [10]. Future research in this area will have important implications for the development of updated diagnostic criteria and the comprehensive treatment of FM patients [11].

\section{Counterpoint: Dr Goldenberg}

The significance of Ge and colleagues' study is tempered by concerns with the validity of MTPs [1]. There is no widely agreed-upon definition of MTPs. Ge and colleagues used the Travell and Simons' criteria, as noted by Bennett. Tough and colleagues, however, found 19 different diagnostic criteria for MTP pain in an extensive literature review [12]. Most of those studies cited the work by Travell and Simons yet failed to apply their diagnostic criteria. The systematic review by Lucas and colleagues concluded: 'On the basis of the limited number of studies available, and significant problems with their design, reporting, statistical integrity, and clinical applicability, physical examination cannot currently be recommended as a reliable test for the diagnosis of trigger points' [13].

There is significant interobserver variability in the MTP examination. For example, four rheumatologists, including Bennett and myself, and four experts on MFP syndrome performed trigger point and tender point examinations on three groups of subjects (seven patients with FM, eight patients with MFP, and eight healthy persons) while blinded as regards diagnosis [14]. Active MTPs were found in $18 \%$ of patients with FM and MFP, but latent trigger points were rare in all groups. Taut muscle bands and muscle twitches were common (50\% and 30\%, respectively) and were noted equally in all three diagnostic groups. There were significant problems with interobserver reliability for taut bands, muscle twitch and active trigger points. The interexaminer reproducibility of the MTP examination varies even among experts but improves with a standardized technique and experience $[15,16]$. Palpation of taut bands and muscle-snapping techniques are especially prone to interobserver variability.

MFP experts point to electrophysiologic evidence of muscle pathology. Ge and colleagues report that EMG evidence of spontaneous electrical activity is the only electrophysiological method to document the existence of MTP, and they therefore used this technique [1]. In their study, the EMG was performed after the manual examination, the needle was 'redirected twice if the first insertion failed to find the spontaneous electrical activity' and the needle electrode length varied with different muscles. Some investigators have been unable to find characteristic spontaneous EMG activity in MTPs [17]. Other techniques said to demonstrate abnormalities in the MTP, such as microdialysis, magnetic resonance elastography and specialized ultrasound, are not widely available and the results have not been duplicated.

Although MFP is considered a localized muscle pain disorder, there is considerable clinical overlap with FM. Two studies reported that 25 to $42 \%$ of subjects with chronic cervical MFP met diagnostic criteria for FM $[18,19]$, and two reports found that 75 to $80 \%$ of FM patients met the criteria for MFP $[19,20]$.

There is strong evidence that abnormal central pain processing, characteristic of $\mathrm{FM}$, is also prominent in MFP. Similar somatosensory pain profiles are found in both FM and MFP [21], and women with MFP had bilateral widespread mechanical pain sensitivity [22]. Bennett mentioned above that sustained mechanical stimulation of latent MTPs induced central sensitization in healthy subjects $[14,15]$. What makes that different from mechanical pressure on tender points inducing central pain? Both Bennett and Ge and colleagues mention that proinflammatory mediators have been reported in MTPs. Similar observations have been found in FM. De Stefano and colleagues found evidence for elevated substance-P immunoreactivity in both MFP and FM [23].

MFP is postulated to be typically self-limited whereas FM is postulated as chronic. FM patients are said to have greater co-morbidity and other somatic symptoms, such as fatigue and sleep and mood disturbances. This 
hypothesis, however, has not been carefully evaluated. MFP experts claim that localized therapy, particularly trigger point injections, are very effective for MTPs but not for tender points. Unfortunately, there are no randomized, controlled studies to document this belief. The uncontrolled studies of multiple different injection techniques, different injectable agents, dry needling and physical modalities attest to lack of universal success. A large, multicenter prospective study comparing subjects who meet criteria for FM, for MFP and for both conditions would be necessary.

Finally, there is no convincing evidence that the MTP can be clinically or pathophysiolgically distinguished from a FM tender point. No study has matched painful muscles containing only tender points with those containing only trigger points. Since trigger points always have a tender point, such a study seems impossible.

Just like fibrositis and fibositic nodules have become historical curiosities, MTPs will eventually be discounted as discrete pathologic abnormalities in the muscle. MFP will be brought into the realm of central pain disorders, including chronic headaches, irritable bowel syndrome, temporomandibular dysfunction and FM. The likelihood that MFP will spread to FM will be attributed to central factors, such as generalized pain tolerance, co-morbid illness and psychosocial factors. Identifying and treating any peripheral pain is a noble pursuit in the management of central pain disorders, such as FM. However, it is unlikely that the MTP is a specific peripheral pain phenomenon.

\section{Abbreviations}

EMG, electromyography; FM, fibromyalgia; MFP, myofascial pain; MTP, myofascial trigger point.

\section{Competing interests}

The authors declare that they have no competing interests.

\section{Author details}

'Fibromyalgia Research Unit, Oregon Health \& Science University, 3455 SW Veterans Road, Portland, OR 97239, USA. ${ }^{2} 2000$ Washington St, Newton, MA 02462 , USA

\section{Published: 30 June 2011}

\section{References}

1. Ge HY, Wang Y, Fernandez-de-Las-Penas C, Graven-Nielsen T, DanneskioldSamsoe B, Arendt-Nielsen L: Reproduction of overall spontaneous pain pattern by manual stimulation of active myofascial trigger points in fibromyalgia patients. Arthritis Res Ther 2011, 13:R48.

2. Travell JG, Simons DG: Myofascial Pain and Dysfunction: The Trigger Point Manual. Baltimore, MD: Williams \& Wilkins; 1983:59-63.

3. Ge HY, Wang Y, Danneskiold-Samsoe B, Graven-Nielsen T, Arendt-Nielsen L: The predetermined sites of examination for tender points in fibromyalgia syndrome are frequently associated with myofascial trigger points. J Pain 2010, 11:644-651.

4. Borg-Stein J, Stein J: Trigger points and tender points: one and the same? Does injection treatment help? Rheum Dis Clin North Am 1996, 22:305-322.

5. Bennett R: Myofascial pain syndromes and their evaluation. Best Pract Res Clin Rheumatol 2007, 21:427-445.
6. Meyer HP: Myofascial pain syndrome and its suggested role in the pathogenesis and treatment of fibromyalgia syndrome. Curr Pain Headache Rep 2002, 6:274-283

7. Lucas N, Macaskill P, Irwig L, Moran R, Bogduk N: Reliability of physical examination for diagnosis of myofascial trigger points: a systematic review of the literature. Clin J Pain 2009, 25:80-89.

8. Ge HY, Fernandez-de-Las-Penas C, Yue SW: Myofascial trigger points: spontaneous electrical activity and its consequences for pain induction and propagation. Chin Med 2011, 6:13.

9. Affaitati G, Costantini R, Fabrizio A, Lapenna D, Tafuri E, Giamberardino MA Effects of treatment of peripheral pain generators in fibromyalgia patients. Eur J Pain 2011, 15:61-69.

10. Alonso-Blanco C, Fernandez-de-Las-Penas C, Morales-Cabezas M, ZarcoMoreno P, Ge HY, Florez-Garcia M: Multiple active myofascial trigger points reproduce the overall spontaneous pain pattern in women with fibromyalgia and are related to widespread mechanical hypersensitivity. Clin J Pain 2011, 27:405-413.

11. Bennett R: Fibromyalgia: present to future. Curr Rheumatol Rep 2005, 7:371-376

12. Tough EA, White AR, Richards S, Campbell J: Variability of criteria used to diagnose myofascial trigger point pain syndrome - evidence from a review of the literature. Clin J Pain 2007, 23:278-286.

13. Lucas N, Macaskill P, Irwig L, Moran R, Bogduk N: Reliability of physical examination for diagnosis of myofascial trigger points: a systematic review of the literature. Clin J Pain 2009, 25:80-89.

14. Wolfe F, Simons DG, Fricton J, Bennett RM, Goldenberg DL, Gerwin R, Hathaway D, McCain GA, Russell IJ, Sanders HO, Skootsky SA: The fibromyalgia and myofascial pain syndromes: a preliminary study of tender points and trigger points in persons with fibromyalgia, myofascial pain syndrome and no disease. J Rheumato/ 1992, 19:944-951.

15. Myburgh C, Lauridsen HH, Larsen AH, Hartvigsen J: Standardized manual palpation of myofascial trigger points in relation to neck/shoulder pain; the influence of clinical experience on inter-examiner reproducibility. Man Ther 2011, 16:136-140.

16. Myburgh $\mathrm{C}$, Larsen $\mathrm{AH}$, Hartvigsen J: A systematic, critical review of manual palpation for identifying myofascial trigger points: evidence and clinical significance. Arch Phys Med Rehabil 2008, 89:1169-1176.

17. Couppe C, Torelli P, Fuglsang-Frederiksen A, Andersen KV, Jensen R: Myofascial trigger points are very prevalent in patients with chronic tension-type headache: a double-blinded controlled study. Clin J Pain 2007, 23:23-27.

18. Sahin N, Karatas O, Ozkaya M, Cakmak A, Berker E: Demographics features, clinical findings and functional status in a group of subjects with cervical myofascial pain syndrome. Agri 2008, 20:14-19.

19. Leblebici B, Pektas ZO, Ortancil O, Hurcan EC, Bagis S, Akman MN: Coexistence of fibromyalgia, temporomandibular disorder, and masticatory myofascial pain syndromes. Rheumatol Int 2007, 27:541-544.

20. Plesh $\mathrm{O}$, Wolfe F, Lane $\mathrm{N}$ : The relationship between fibromyalgia and temporomandibular disorders: prevalence and symptom severity. J Rheumatol 1996, 23:1948-1952.

21. Pfau DB, Rolke R, Nickel R, Treede RD, Daublaender M: Somatosensory profiles in subgroups of patients with myogenic temporomandibular disorders and fibromyalgia syndrome. Pain 2009, 147:72-83.

22. Fernandez-de-Las-Penas C, Galan-del-Rio F, Fernandez-Carnero J, Pesquera J, Arendt-Nielsen L, Svensson P: Bilateral widespread mechanical pain sensitivity in women with myofascial temporomandibular disorder: evidence of impairment in central nociceptive processing. J Pain 2009, 10:1170-1178.

23. De Stefano R, Selvi E, Villanova M, Frati E, Manganelli S, Franceschini E, Biasi G, Marcolongo R: Image analysis quantification of sustance $P$ immunoreactivity in the trapezius muscle of patients with fibromyalgia and myofascial pain syndrome. J Rheumatol 2000, 27:2906-2910.

doi:10.1186/ar3357

Cite this article as: Bennett RM, Goldenberg DL: Fibromyalgia, myofascial pain, tender points and trigger points: splitting or lumping? Arthritis Research \& Therapy 2011, 13:117. 